\title{
Combination of intramedullary rod, wrapping bone grafting and llizarov's fixator for the treatment of Crawford type IV congenital pseudarthrosis of the tibia: mid-term follow up of 56 cases
}

\author{
Guang-hui Zhu, Hai-bo Mei, Rong-guo He, Yao-xi Liu, Kun Liu, Jin Tang and Jiang-yan Wu
}

\begin{abstract}
Background: The purpose of this study was to investigate the initial union rate, refracture rate and residual deformities of congenital pseudarthrosis of the tibia (CPT), using combined surgery including pseudarthrosis resection, intramedullary rodding, autogenous iliac bone grafting and llizarov's fixator, with a mean 5.2 years follow-up.

Methods: We retrospectively reviewed the records and diagrams of patients with Crawford type IV congenital pseudarthrosis of the tibia between February 2007 and March 2010. Patients managed by pseudarthrosis resection, intramedullary rod of the tibia, wrapping autogenous iliac bone grafting and llizarov's fixator were enrolled. We evaluated the bone union rate, tibial alignment, limb length discrepancy (LLD), valgus deformity of the ankle and the frequencies of refracture during period of follow-up.

Results: There were 56 cases enrolled in the study, with a mean follow-up 5.2 years (range, 3 to 6.7 years). The mean age of the patients at surgery was 3.5 years (range, 1.5 to 12.4 years). Fifty ( $89.2 \%$ ) of the 56 patients had primary bone union at site of pseudarthrosis, while 5 obtained union after second surgery and 1 failed. The average time spent to obtain pseudarthrosis initial union was 4.5 months (range, 3.0 to 10.0 months) and mean duration of llizarov treatment was 4.7 months (range, 3.2 to 10.4 months). Eleven (19.6\%) patients had proximal tibial valgus with a mean angle of 9. $5^{\circ}$ (range, 5 to $24^{\circ}$ ), while $10\left(17.9 \%\right.$ ) patients had ankle valgus deformities with a mean of $12.3^{\circ}$ (range, 6 to $21^{\circ}$ ). Sixteen (28.6\%) patients had an average $2.2 \mathrm{~cm} \mathrm{LLD} \mathrm{(range,} 1.5-4.2 \mathrm{~cm}$ ). Of the 50 cases who obtained initial bone union of pseudarthrosis, 13 (26.0 \%) had refracture which need cast immobilization or secondary surgery.
\end{abstract}

Conclusions: This combined surgery obtained initial union rate of $89.2 \%$ at primary surgery while the refracture rate is $26.0 \%$. However, residual deformities such as proximal tibial valgus, LLD and ankle valgus were also existed which should be pay more attention to and dealt with.

Trial registration: This study was registered in ClinicalTrials.gov under the name "The Effect of Combined Surgery in Management of Congenital Pseudarthrosis of Tibia" (NCT02640040), which was released on August 31, 2015.

Keywords: Congenital pseudarthrosis of tibia, Intramedullary rodding, llizarov's fixator, Union rate, Residual deformities

* Correspondence: meihaibo8@163.com

Department of Orthopedics, Hunan Children's Hospital, 86 Ziyuan road,

Changsha, Hunan, People's Republic of China

(c) 2016 The Author(s). Open Access This article is distributed under the terms of the Creative Commons Attribution 4.0 International License (http://creativecommons.org/licenses/by/4.0/, which permits unrestricted use, distribution, and reproduction in any medium, provided you give appropriate credit to the original author(s) and the source, provide a link to the Creative Commons license, and indicate if changes were made. The Creative Commons Public Domain Dedication waiver (http://creativecommons.org/publicdomain/zero/1.0/) applies to the data made available in this article, unless otherwise stated. 


\section{Background}

Congenital pseudarthrosis of the tibia (CPT) is a rare disorder and characterized by segmental osseous dysplasia and progression to fracture at walking age with a substantial risk of non-union, leg length discrepancy (LLD), and malalignment of the tibia. CPT is recalcitrant to standard treatment and patients with this disorder often require multiple surgical procedures in an effort to achieve bone union and a functional extremity [1-3]. Currently, three surgical techniques have been most commonly used, including vascularized fibular graft, intramedullary rod stabilization associated autogenous bone-grafting or bone morphogenetic protein-2, and Ilizarov technique, obtaining markedly improved primary union rate of CPT (range, 28 to $92 \%$ ) [4-7]. With vascularized fibular graft, pseudarthrosis union can be achieved in most patients; however the microsurgery techniques has a long learning curve. Intramedullary rod has been widely used for treatment of CPT to obtain union and protect against refracture, but it is an unstable fixation of the distal tibial fragment, and may cause ankle stiffness and arthritic changes in the ankle joint [8-10]. The Ilizarov technique has a high rate of fusion, allowing for the correction of axial malalignment and leg shortening at the same procedure. However, there is a risk of refracture after removal of the fixator [10-12].

Most recently, a tendency to treat CPT using combined Ilizarov's technique and intramedullary rod were evolved as being more reliable in achieving and maintaining union, diminishing residual deformities such as valgus angulations, leg-length discrepancy, and refracture [13-15]. Since February 2007, we used a combined surgical technique, including pseudarthrosis resection, intramedullary rod of the tibia, wrapping autogenous iliac bone graft and Ilizarov's technique for the treatment of congenital pseudarthrosis of the tibia, to manage Crawford IV congenital pseudarthrosis of the tibia.

The purpose of this study was to present our result of combined surgical technique in management of congenital pseudarthrosis of the tibia and investigate the frequency of re-fracture and residual deformities during a mean 5.2 years follow-up.

\section{Methods}

Between February 2007 and March 2010, 56 consecutive cases with Crawford type IV congenital pseudarthrosis of the tibia managed by pseudarthrosis resection, intramedullary rod of the tibia, wrapping autogenous iliac bone grafting and Ilizarov's fixator, were retrospectively reviewed.

There were 43 boys and 13 girls with the right leg involved in 30 patients, left in 26. Pseudarthrosis was at the lower third of the tibia in 41 patients, middle third of diaphysis in 12 patients, and upper third in the other 3 patients. Forty-eight patients $(85.7 \%)$ presented with neurofibromatosis type 1 (NF1) and 8 patients (14.3\%) with unknown etiology. Forty-one patients $(73.2 \%)$ had no previous surgery, while 15 patients (26.8\%) underwent 1 to 4 unsuccessful procedures in other hospital prior to referral to our institution. The mean age at the initial operation was 3.5 years (range, 1.5 to 12.4 years). The average limb-length discrepancy (LLD) pre-operatively was $1.6 \mathrm{~cm}$ (range, 1.0 to $3.8 \mathrm{~cm}$ ).

\section{Surgical technique Harvesting autogenic iliac bone}

The patient was placed in a supine position on an operating table. The iliac bone graft was harvested through a straight incision centered over the anterior superior iliac spine. The apophysis of the ilium was split and the outer table of the anterolateral surface of the ilium was exposed subperiosteally. A rectangular cortex was obtained from the outer table of the ilium and as much cancellous bone as possible was curetted from the supra-acetabular region, while keeping the inner wall intact. Several holes were made in the rectangular cortex with $1.5 \mathrm{~mm}$ Kirschner wire and then it was woven sutured with absorbable sutures on each corner and bent to cylindrical shaped (Fig. 1).

\section{Excision of pseudarthrosis, intramedullary rod insertion, and installation of llizarov's fixator}

A tourniquet was used to decrease the blood loss. An anterior straight incision over the site of the pseudarthrosis was made to expose the pseudarthrosis. The deep fascia of the anterior compartment was routinely divided longitudinally to prevent compartment syndrome. Excision of the pseudarthrosis included the abnormal periosteum, surrounding pathologic soft tissues and the sclerotic bone ends until normal tissue planes are encountered. The medullary canal of both proximal and distal tibial fragment was opened with sequentially larger drill until a $4 \mathrm{~mm}$ or $4.5 \mathrm{~mm}$ intramedullary rod could be inserted. In patients with concomitant fibular pseudarthrosis, fibrous tissue at site of pseudarthrosis of the fibula was also resected and typically fixed with a $1.6-1.8 \mathrm{~mm}$ intramedullary Kirschner wire based on the age of patient and the diameter of the fibula. But in patients with intact fibular, because an intact fibula will hinder the apposition of the tibial fragments, osteotomy of the proximal fibula was performed to allow complete apposition of the ends of the tibia, but the fibula osteotomy was not fixated but to leave both segments overlapped.

A Williams' rod of appropriated length and diameter determined on digital radiography preoperatively was inserted into the medullary canal of the tibia across the CPT site, from proximal to distal direction via the calcaneus and talus and out of the sole through the heel pad. The fluoroscopy was used to ensure that the rod was located in the center of distal tibial physis on anteroposterior and 

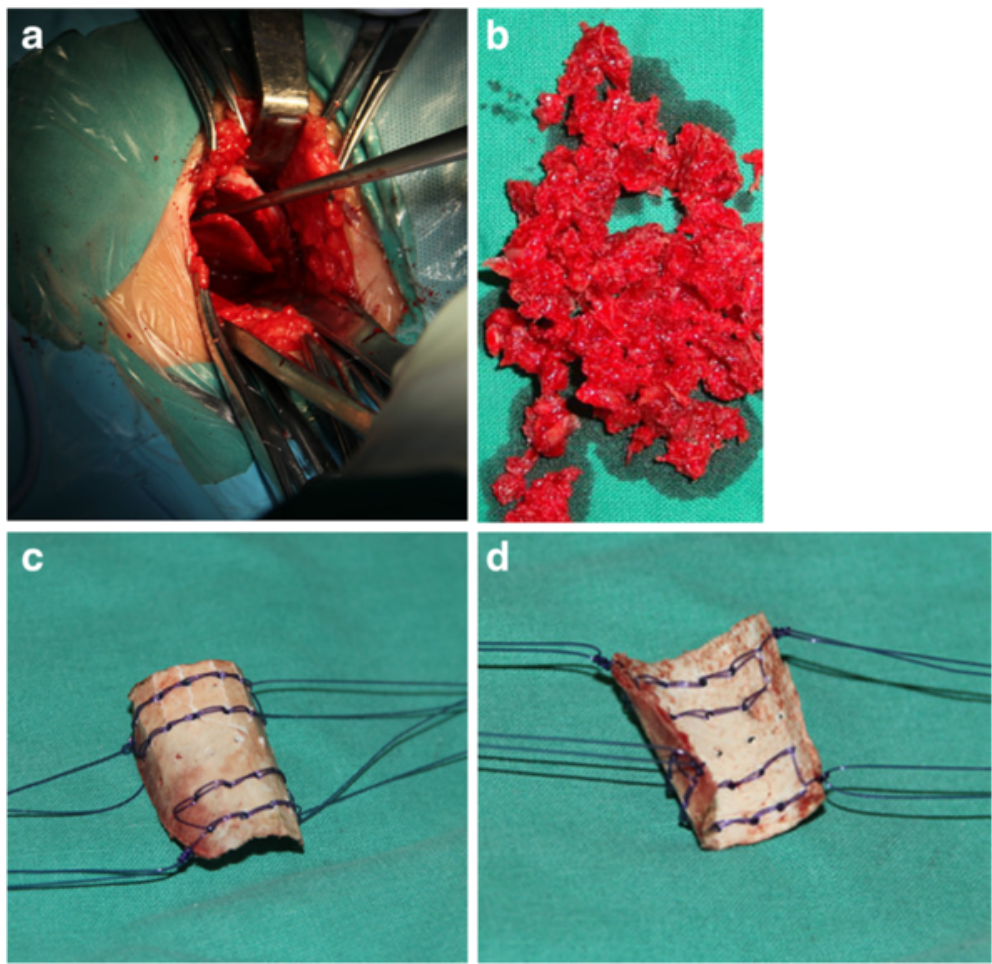

Fig. 1 Harvesting and suturing autogenic iliac bone. Exposure of the outer table of the ilium, harvesting a rectangular cortex (a). Cancellous bone curetted from supra-acetabular region (b). Holes were made in the rectangular cortex with Kirschner wire and with doubled absorbable sutures on each corner (c). The rectangular cortex was bent to produce a cylindrical shape for wrapping the cancellous bone graft. It would have greenstick fracture on the cortex (d)

lateral views and that a neutral dorsiflexion-plantar flexion of the foot and neutral varus-valgus alignment of the ankle were maintained. The rod was then driven retrograde into the proximal tibial fragment, which was anatomically aligned in both the coronal and the sagittal planes was verified using intraoperative imaging.

After finishing the insertion of the intramedullary rod, the Ilizarov's fixator was mounted with one full ring above the site of pseudarthrosis and one below. Both rings were fixed with two or three 1.5 or $2.0 \mathrm{~mm}$ tensioned Kirschner wires through the tibia and were connected together by threaded rods, subsequently applying appropriate pressure at site of pseudarthrosis of the tibia. If the distal tibial segment was less than $3 \mathrm{~cm}$ long, a calcaneal half-ring or U-shaped ring was used to increase distal stability. In addition, if the tibial length discrepancy was more than $3 \mathrm{~cm}$ compared with the normal side of the tibia and the age of patient was more than 3 years, proximal tibial lengthening was performed concomitantly through a proximal metaphysic corticotomy by distraction osteogenesis at initial operation. Another ring was added proximal to the metaphysic corticotomy site. The bone segments proximal and distal to the corticotomy site were fixed with three 1.5 or $2.0 \mathrm{~mm}$ Kirschner wires connected to the ring (Fig. 2a).

\section{Wrapping autogenic iliac bone graft}

The previously harvested the cylindrical cortex was wrapped around site of pseudarthrosis of the tibia after the application of the Ilizarov fixator. The cancellous bone grafts were placed circumferentially between the grafted cortex and site of pseudarthrosis and the cortex was tied with absorbable sutures, which had been connected to the cortex previously, establishing a sealing environment for enhancement of osteogenesis (Fig. 2b and c).

\section{Postoperative management}

The wound was sutured routinely with retention of a drainage tube. The drainage tube was usually removed within $72 \mathrm{~h}$ after surgery. For cases with proximal tibial lengthening, distraction osteogenesis was started 1 week post-operation, and the speed for bone transportation was $0.5 \mathrm{~mm} /$ day in 4 increments [13]. The patients were followed every 4 weeks for the first 3 months and every 6 weeks for the next 3 months until radiographic union was obtained. When the pseudarthrosis of the tibia had consolidated, the Ilizarov's fixator was removed and a short leg cast was applied for an average of 2 months. After the cast is removed, the patient was then fit with a protective knee-ankle-foot orthosis and was allowed to begin weight-bearing. Hereafter, patients were followed 

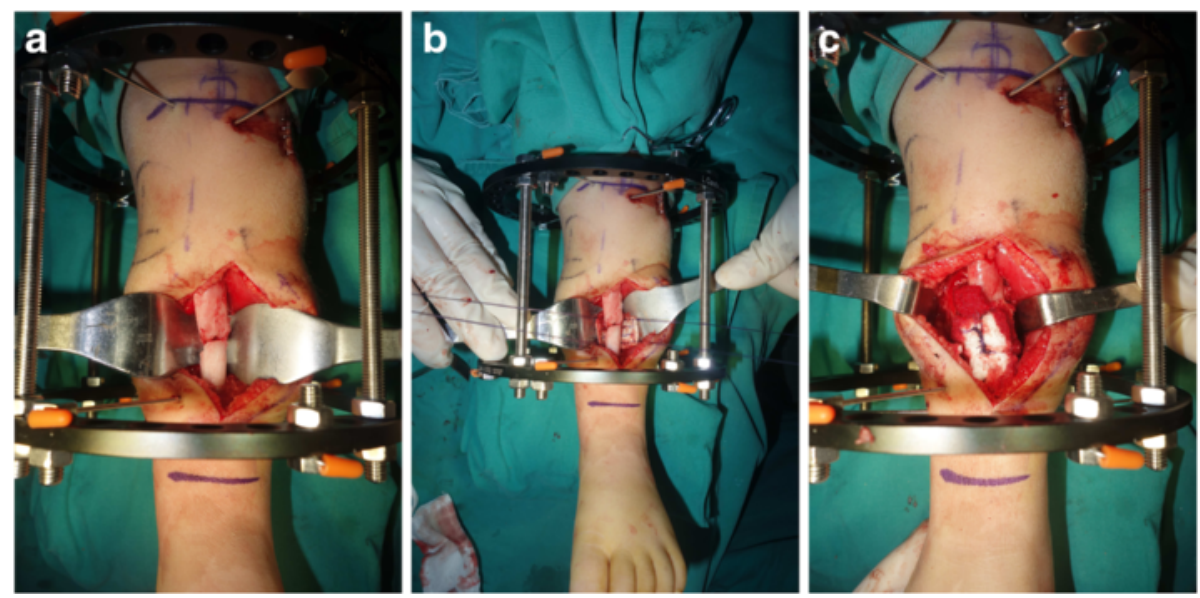

Fig. 2 Installation of llizarov's fixator and wrapping bone grafting. Resection of pseudarthrosis and installation of llizarov fixator (a). Cancellous bone compacted circumferentially between the cylindrical cortex and the pseudarthrosis of the tibia (b). The wrapping bone graft was secured by tying the sutures (c)

at interval of 3 months until the patient reaches skeletal maturity.

In order to minimize the duration of ankle joint immobilization and the potential damage of the articular cartilage with prolonged ankle transfixation of the intramedullary rod of the tibia, the rod across the ankle joint was surgically pushed into epiphysis of the distal tibia at 18 to 24 months after union of pseudarthrosis, using a $2.5 \mathrm{~mm} \mathrm{~K}$-wire or another rod with threaded end to reach or connect the hollow threaded end of Williams' rod, and drive the rod into the epiphysis of the distal tibia under fluoroscopy.

\section{Evaluation}

Sequential radiographs taken during follow-up and clinical chart of each patient were reviewed to evaluate outcome in patient with congenital pseudarthrosis of the tibia treated by this combined surgical technique, including time taken for union at the site of pseudarthrosis, tibial alignment, limb length discrepancy, the status of fibula, and frequency of re-fracture during duration of follow-up.

Union of congenital pseudarthrosis of the tibia was defined as evidence of bridging callus on four cortices across the transverse tibial cortical defects without visible fracture line on both the anteroposterior and lateral radiographs [16].

The assessment of tibial alignment consisted of measurements of the proximal tibial valgus and ankle valgus on anterioposterior radiograph. The measurement of proximal tibial valgus was created by the intersection of a line parallel to proximal physis and another line along the axis of proximal third of diaphyseal in the tibia. Ankle valgus was assessed by tibiotalar angle that was measured at the intersection of the mid-diaphyseal line of the tibia and a line drawn across the flat subchondral line of the talar dome (Fig. 3). If patient who had $>3^{\circ}$ of proximal tibial valgus and $>5^{\circ}$ of ankle valgus was defined as proximal tibial valgus and ankle valgus deformity respectively.

The length of involved tibia and contralateral tibia were measured directly from proximal physis to distal physis of tibia on digital X-ray imaging.

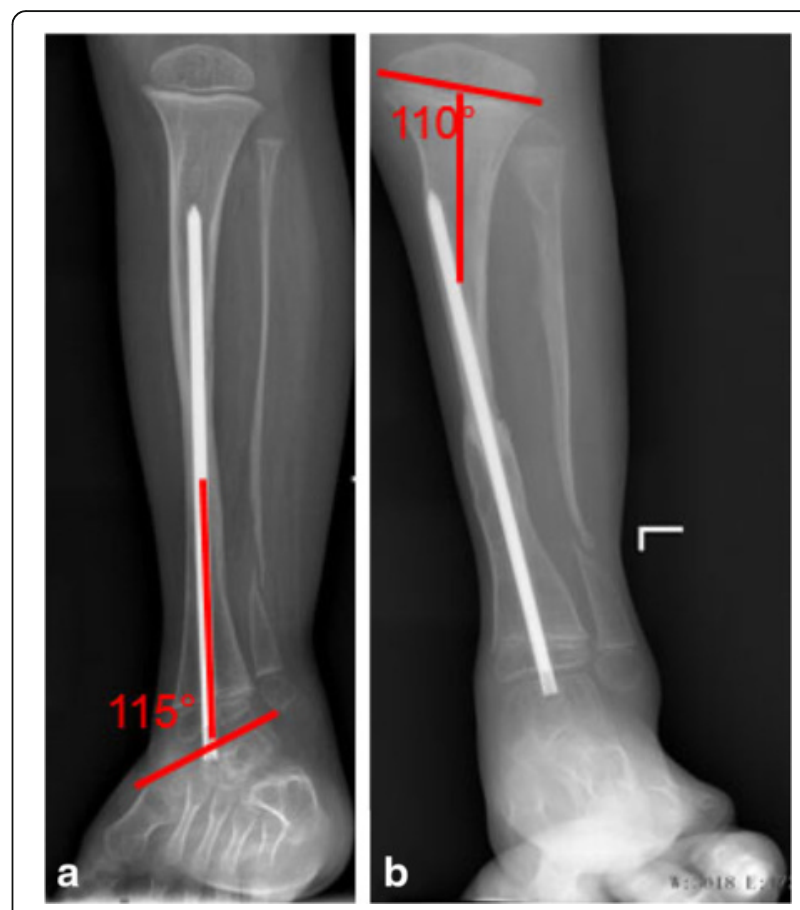

Fig. 3 The evaluation on X-ray. Illustrations of the measurements of ankle valgus (a) and proximal tibial valgus (b) respectively 
Refracture was defined as an obvious fracture on a plain radiograph following the primary union at site of pseudarthrosis.

\section{Results}

There were 56 patients with congenital pseudarthrosis of the tibia treated by our combined procedure enrolled. The mean follow-up was 5.2 years (range, 3 to 6.7 years). Fifty (89.2\%) of the 56 patients had initial union of pseudarthrosis of the tibia. There were 6 cases that did not obtained initial union. Among the 6 cases, 3 achieved union after another attempt of wrapping autogenic iliac bone graft and Ilizarov fixator compression, 2 obtained final union using the Masquelet technique, only one failed union after second surgery (Masquelet technique) and refused to subsequent treatment. The average time spent to obtain initial union was 4.5 months (range, 3.0 to 10.0 months) and mean duration of Ilizarov treatment was 4.7 months (range, 3.2 to 10.4 months).

At recent follow-up, 12 patients had the rod being located in the epiphysis of the distal tibia with growth of the tibia, 4 patients with the rod in the tibia medullary cavity, while the other 40 patients had underwent a surgery to push the intramedullary rod to the epiphysis of the distal tibia.

Eleven (19.6\%) patients had proximal tibial valgus with a mean angle of $9.5^{\circ}$ (range, 5 to $24^{\circ}$ ). Ten (17.9\%) patients had ankle valgus deformities with a mean of $12.3^{\circ}$ (range, 6 to $21^{\circ}$ ). Sixteen $(28.6 \%$ ) patients had an average $2.2 \mathrm{~cm}$ LLD (range, 1.5-4.2 cm).

Of the 50 cases who obtained initial bone union of pseudarthrosis, $13(26.0 \%)$ had refracture which need cast immobilization or secondary surgery. Ten patients sustained re-fracture at the original pseudarthrosis site, 2 patients at a more proximal site, and one patient at a more distal site. Patients with re-fracture were treated with cast immobilization firstly. If union was not obtained, surgical management was considered. Among the 13 patients with refracture, 8 patients healed at refracture site by cast immobilization, 3 patients obtained solild union by Ilizarov fixator to compress at the refracture site, but the remaining 2 patients failed to retrieve bony union with Ilizarov compression.

Ankle joint stiffness was identified in 4 patients due to the previous failed surgery. There were 11 cases with pin-tract infection which was cured by pin-tract nurse and oral administration of antibiotics. Two cases developed central epiphyso-metaphyseal bone bridge formation, however no growth disturbance was found. There were no other complications such as knee contracture, neurovascular compromise, peroneal nerve injury, or compartment syndrome in any patient.

The demographic data of results were summarized in Table 1 . We present a typical case in Fig. 4.
Table 1 Patients result

\begin{tabular}{ll}
\hline Parameters & \\
\hline Mean age at index surgery & 3.5 years (range, 1.5 to 12.4 years) \\
Follow up & 5.2 years (range, 3 to 6.7 years) \\
Bone initial union time & 50 cases (89.2 \%) \\
& 4.5 months (range, 3.0 to 10.0 months) \\
& 11 cases (19.6 \%) \\
Proximal tibial valgus & $9.5^{\circ}$ (range, 5 to $\left.24^{\circ}\right)$ \\
& 10 cases $(17.9 \%)$ \\
Ankle valgus & $12.3^{\circ}$ (range, 6 to $\left.21^{\circ}\right)$ \\
& 16 cases $(28.6 \%)$ \\
Limb-length discrepancy & $2.2 \mathrm{~cm}$ (range, $1.5-4.2 \mathrm{~cm})$ \\
& 13 cases (26.0 \%) \\
\hline
\end{tabular}

\section{Discussion}

Although the outcomes from numerous treatment methods for congenital pseudarthrosis of the tibia had shown greatly improvement in primary union $[1,5]$, there are still many challenging problems that include potential risks of non-union, re-fractures, leg length discrepancy (LLD), and malalignment of the tibia and the ankle [17]. Currently, there is no consensus on the optimal surgical technique for treatment of congenital pseudarthrosis of the tibia.

Ilizarov technique was widely used to manage with CPT, with the biggest advantage of the tibial fragments being compressed, lengthening of the tibia, and correction of angular deformities concomitantly. The drawback of Ilizarov technique was its lack of protection against re-fractures after removal of the fixator and usually associated with a high re-fracture rate $[4,12]$. Inan et al. reported 16 patients treated with Ilizarov, all the patients united but 10 patients had late axial malalignment and re-fracture [17]. Cho et al. reported a rate of re-fracture of $47 \%$ in a series of 23 patients with atrophic CPT treated by Ilizarov's technique [18].

Intramedullary rod was another commonly method used for treatment of CPT popularized by Umber et al. in 1982 and obtained relative high union rate $[5,19]$. An additional benefit of this technique could provide protection against re-fracture before skeletal maturity $[1,20]$. Dobbs successfully treated 18 of 21 patients with intramedullary rod but re-fracture occurred in twelve patients [9]. Joseph and Mathew treated 14 patients with congenital pseudarthrosis of the tibia using excision of the pseudarthrosis, double onlay autogenous cortical bone grafting, and intramedullary nailing [21]. Of 14 pseudarthrosis of the tibia, 12 patients achieved union without re-fractures occurred at the site of the original pseudarthrosis. But in practice, we found the onlay cortical bone grafting to be unstable and may be absorbed spontaneously during the fixation. Thus, since February 2007, we used intramedullary rod and wrapping 

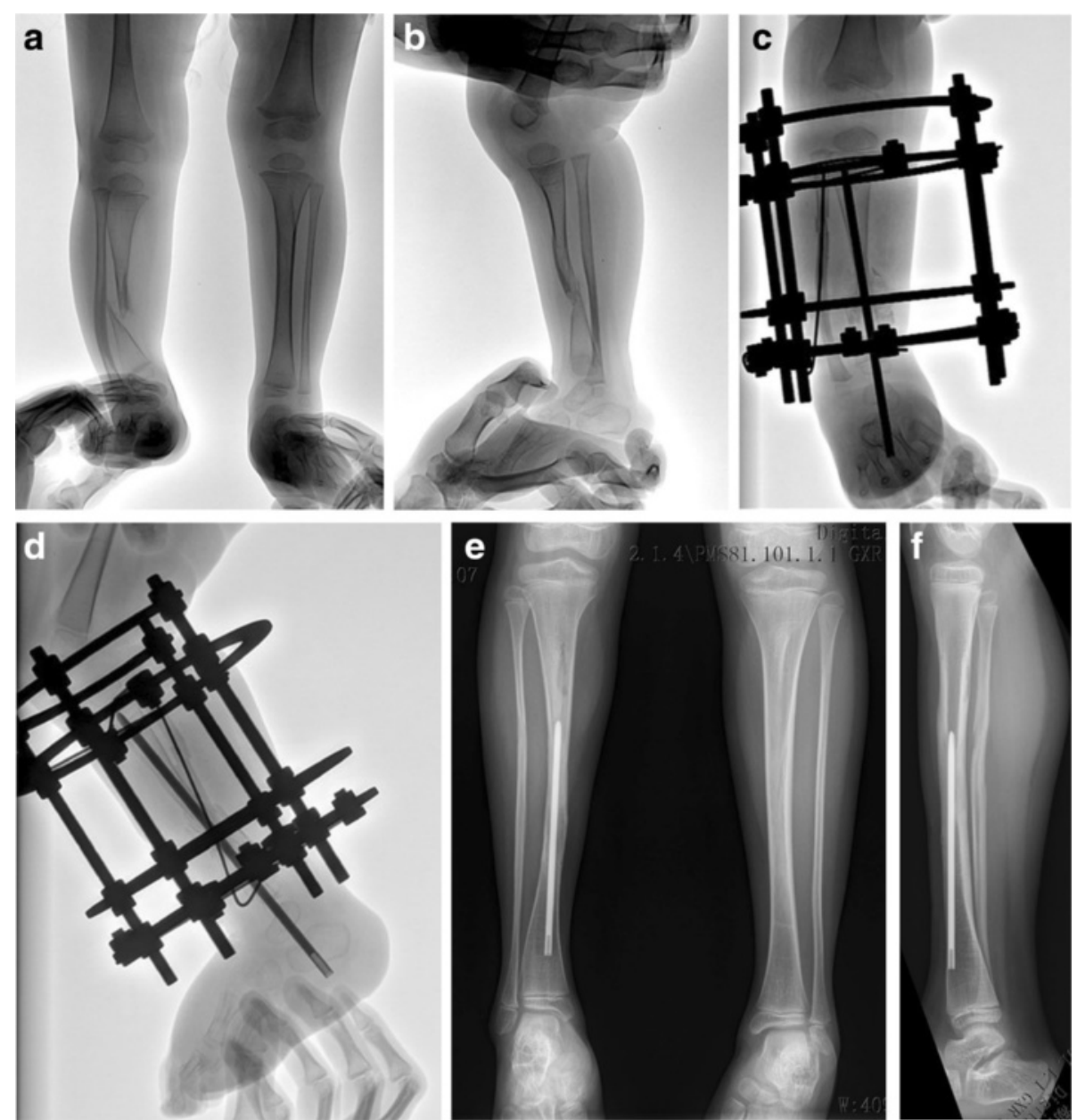

Fig. 4 A typical case of 2.5 years boy with congenital pseudarthrosis of the tibia. Preoperative anteroposterior and lateral X-rays shows Crawford type IV CPT and intact fibula $(\mathbf{a}, \mathbf{b})$. Anteroposterior and lateral radiograph of the same patient taken at 2 months after combined surgey (c, $\mathbf{d})$. X-ray of 5.4 years post operation shows solid union of the pseudarthrosis with good alignment of the tibia without ankle valgus, tibia angulation or LLD. The rod was in the tibia medullary cavity with growth without surgical pushing of the rod $(\mathbf{e}, \mathbf{f})$

autogenic iliac bone graft in combination with Ilizarov's fixator for treatment of congenital pseudarthrosis of the tibia and attempted to take advantages of both Ilizarov's fixator and intramedullary fixation, and avoid the disadvantage of onlay cortical bone grafting. The Ilizarov's fixator could provide high fusion rate with good alignment control, while intramedullary fixation could stabilize the pseudarthrosis and avoid re-fracture. The wrapping autogenous cortex and cancellous bone grafting would make the grafting and pseudarthrosis close contacted over a large surface area, which may enhance mechanical stability and provide a unique biologic environment promoting union.

In current study, 56 patients with congenital pseudarthrosis of the tibia were treated by the combined procedure and $50(89.3 \%)$ patients achieved initial union at site of pseudarthrosis at a mean follow-up of 5.2 years, while another 5 case obtained union after second surgery, only 1 failed union. During a 5.2 years follow up (the shortest was 3.5 years), we observed the total refracture rate was $26.0 \%$. The primary union rate and the refracture rate of ours were comparable to those reported in the literature $[8,21,22]$. However, high frequency of residual deformities including proximal tibial valgus, ankle valgus, LLD and still existed, which is similar to Dobbs and Kristiansen's study [9, 12].

The incidence of proximal tibial valgus post opreration was $19.6 \%$ with an average of $9.5^{\circ}$, and we observed proximal tibial dysplasia in these patients. Cho et al. pointed that the proximal tibial dysplasia reflects a pathological process of the periosteum same as fibrous hamartoma in the pseudarthrosis site, and the extent of this lesion varies in patients [23]. We surmise that proximal tibial dysplasia may result to unbalanced growth of proximal tibial which seems to be attributable to tibial 
valgus of our series. Epiphysiodesis by staple or screw fixation can be introduced to correct tibial valgus in these younger patients.

Ten (17.9 \%) patients had ankle valgus deformities post operation with a mean of $12.3^{\circ}$, which is similar to other investigators $[9,10]$. Some authors reported that persistent fibular pseudarthrosis may relate to progressive ankle valgus $[12,17]$. Distal tibia corrective osteotomy, fibular stabilization by temporary hemiepiphysiodesis or tibiofibular synostosis would be advocated if serious ankle valgus deformities were existed.

An average of $2.2 \mathrm{~cm}$ LLD in 16/56 case was observed in our series. The LLD following success union may ascribe to the discrepancy of limb length pre-operation and the acute shortening at the time of the pseudarthrosis resection. Either epiphysiodesis of the contralateral side or tibial lengthening of the involved side could be used to equalize the limb length [12, 17]. In our experience, we suggested that proximal tibial lengthening could be considered when the LLD was more than $4 \mathrm{~cm}$ in younger children after primary union of CPT [13].

Residual problems such as proximal tibia valgus, ankle valgus, LLD and refracture are common despite successful union of pseudoarthrosis, thus more attention should be paid to these residual deformities and revision surgeries may need to correct these deformities.

The present study has three limitations. First, it was not long enough to document real incidence of residual deformities with this combined approach, including proximal tibial valgus, ankle valgus, LLD, and refracture. It is necessary to follow up these younger patients until skeletal maturity and to evaluate the long term outcome of the combined technique. Second, we need to carry out some well designed prospective studies to investigate the advantage of wrapping autogenic iliac bone graft. Last but not least, due to its retrospective properties, some data were not available to carry out comparative research for this series.

\section{Conclusion}

This combined technique has advantages of both allowing tibial fragments being compressed at site of pseudarthrosis of the tibia to facilitate bony union and providing protection against re-fracture. However, residual deformities such as proximal tibial valgus, LLD and ankle valgus were also existed which should be pay more attention to and dealt with. It is necessary to follow up until skeletal maturity and to evaluate the long term outcome of the combined technique.

\section{Abbreviations}

CPT: Congenital pseudarthrosis of the tibia; LLD: Limb length discrepancy; NF1: neurofibromatosis type 1

\section{Acknowledgements}

The authors would like to thank all participating patients, as well as all the co-investigators, and colleagues who made this study possible.

\section{Funding}

The study was not supported by any funding.

\section{Availability of data and materials}

The data supporting the conclusions of this article is stored at the Data and File Room of orthopedics department, Hunan Children's Hospital. The investigator in charge is HM. Data will not be shared now in an open access repository because this study is part of research which is registered in ClinicalTrials.gov (ClinicalTrials.gov Identifier: NCT02640040), and we have not finished the data analysis yet. If anyone is interested in exploring specific issue, please contact HM.

\section{Authors' contributions}

GZ accountable for the integrity and analysis of the data, and the writing of the manuscript, HM accountable for the conception and execution of the research. $\mathrm{RH}$ accountable for the execution of the research, $\mathrm{KL}$ accountable for the execution of the research, JT and $\mathrm{YL}$ accountable for the analysis and interpretation of data, JW accountable for the conception and execution of the research. All authors read and approved the final manuscript.

\section{Competing interests}

The authors declare that they have no competing interests.

\section{Consent for publication}

We have obtained written consent from the guardian of the patients to publish the pictures and clinical data.

Ethics approval and consent to participate

The Ethical review board of Hunan Children's Hospital approved this study and the form of consent. Written informed consent was received from all study participants.

Levels of evidence

Level IV, Therapeutic.

Received: 27 April 2016 Accepted: 13 October 2016

Published online: 22 October 2016

\section{References}

1. Thabet AM, Paley D, Kocaoglu M, Eralp L, Herzenberg JE, Ergin ON. Periosteal grafting for congenital pseudarthrosis of the tibia: a preliminary report. Clin Orthop Relat Res. 2008:466:2981-94.

2. Anderson DJ, Schoenecker PL, Sheridan JJ, Rich MM. Use of an intramedullary rod for the treatment of congenital pseudarthrosis of the tibia. J Bone Joint Surg Am. 1992;74(2):161-8.

3. Lehman WB, Atar D, Feldman DS, Gordon JC, Grant AD. Congenital pseudarthrosis of the tibia. J Pediatr Orthop B. 2000;9:103-7.

4. Grill F, Bollini G, Dungl P, Fixsen J, Hefti F, Ippolito E, Romanus B, Tudisco C, Wientroub S. Treatment approaches for congenital pseudarthrosis of tibia: results of the EPOS multicenter study. European Paediatric Orthopaedic Society (EPOS). J Pediatr Orthop B. 2000;9:75-89.

5. Joseph B, Somaraju W, Shetty SK. Management of congenital pseudarthrosis of the tibia in children under 3 years of age: effect of early surgery on union of the pseudarthrosis and growth of the limb. J Pediatr Orthop. 2003:23(6):740-6.

6. Weiland AJ, Weiss AP, Moore JR, Tolo VT. Vascularized fibular grafts in the treatment of congenital pseudarthrosis of the tibia. J Bone Joint Surg Am. 1990;72:654-62

7. Richards BS, Oetgen ME, Johnston CE. The use of rhBMP-2 for the treatment of congenital pseudarthrosis of the tibia: a case series. J Bone Joint Surg Am. 2010;92:177-85.

8. Shah H, Doddabasappa SN, Joseph B. Congenital pseudarthrosis of the tibia treated with intramedullary rodding and cortical bone grafting: a follow-up study at skeletal maturity. J Pediatr Orthop. 2011:31:79-88.

9. Dobbs MB, Rich MM, Gordon JE, Szymanski DA, Schoenecker PL. Use of an intramedullary rod for treatment of congenital pseudarthrosis of the tibia. A long-term follow-up study. J Bone Joint Surg Am. 2004;86:1186-97. 
10. Choi $\mathrm{H}, \mathrm{Cho} \mathrm{T}-\mathrm{J}, \mathrm{Moon} \mathrm{HJ}$. llizarov treatment of congenital pseudarthrosis of the tibia. A multi-targeted approach using the llizarov technique. Clin Orthop Surg. 2011;3:1-8.

11. Paley D, Catagni M, Argnani F, Prevot J, Bell D, Armstrong P. Treatment of congenital pseudoarthrosis of the tibia using the llizarov technique. Clin Orthop. 1992;280:81-93.

12. Kristiansen LP, Steen $H$, Terjesen T. Residual challenges after healing of congenital pseudarthrosis in the tibia. Clin Orthop Relat Res. 2003;414:228-37.

13. Zhu GH, Mei HB, He RG, Liu K, Tang J, Wu JY. Effect of distraction osteogenesis in patient with tibial shortening after initial union of Congenital Pseudarthrosis of the Tibia(CPT): a preliminary study. BMC Musculoskelet Disord. 2015;16:216.

14. Mathieu L, Vialle R, Thevenin-Lemoine C, Mary P, Damsin JP. Association of llizarov's technique and intramedullary rodding in the treatment of congenital pseudarthrosis of the tibia. J Child Orthop. 2008;2(6):449-55.

15. Agashe MV, Song SH, Refai MA, Park KW, Song HR. Congenital pseudarthrosis of the tibia treated with a combination of llizarov's technique and intramedullary rodding. Acta Orthop. 2012;83(5):515-22

16. Nicolaou N, Ghassemi A, Hill RA. Congenital pseudarthrosis of the tibia: the results of an evolving protocol of management. J Child Orthop. 2013;7:269-76.

17. Inan M, El Rassi G, Riddle EC, Kumar SJ. Residual deformities following successful initial bone union in congenital pseudarthrosis of the tibia. J Pediatr Orthop. 2006;26:393-9.

18. Cho TJ, Choi IH, Lee SM, Chung CY, Yoo WJ, Lee DY, Lee JW. Refracture after llizarov osteosynthesis in atrophic type congenital pseudarthrosis of the tibia. J Bone Joint Surg (Br). 2008:90:488-93.

19. Umber JS, Moss SW, Coleman SS. Surgical treatment of congenital pseudarthrosis of the tibia. Clin Orthop. 1982;166:28-33.

20. Johnston CE. Congenital pseudarthrosis of the tibia: results of technical variations in the charnley-williams procedure. J Bone Joint Surg Am. 2002;84:1799-810.

21. Joseph B, Mathew G. Management of congenital pseudarthrosis of the tibia by excision of the pseudarthrosis, onlay grafting, and intramedullary nailing. J Pediatr Orthop B. 2000;9:16-23.

22. Ohnishi I, Sato W, Matsuyama J, Yajima H, Haga N, Kamegaya M, Minami A, Sato M, Yoshino S, Oki T, Nakamura K. Treatment of congenital pseudarthrosis of the tibia: a multicenter study in Japan. J Pediatr Orthop. 2005;25:219-24

23. Cho TJ, Choi IH, Lee KS, Lee SM, Chung CY, Yoo WJ, Lee DY. Proximal tibial lengthening by distraction osteogenesis in congenital pseudarthrosis of the tibia. J Pediatr Orthop. 2007;27(8):915-20.

\section{Submit your next manuscript to BioMed Central and we will help you at every step:}

- We accept pre-submission inquiries

- Our selector tool helps you to find the most relevant journal

- We provide round the clock customer support

- Convenient online submission

- Thorough peer review

- Inclusion in PubMed and all major indexing services

- Maximum visibility for your research

Submit your manuscript at www.biomedcentral.com/submit

) Biomed Central 